\title{
Sedimentation, trapping, and rectification of dilute bacteria
}

\author{
J. TAILLEUR and M. E. CATES
}

SUPA, School of Physics And Astronomy, University of Edinburgh, JCMB Kings Buildings, Edinburgh EH9 3JZ, United Kingdom

PACS 05.40.-a - Fluctuation phenomena, random processes, noise, and Brownian motion

PACS $87.10 . \mathrm{Mn}-$ Stochastic modeling

PACS $87.17 . \mathrm{J} j-$ Cell locomotion, chemotaxis

\begin{abstract}
The run-and-tumble dynamics of bacteria, as exhibited by E. coli, offers a simple experimental realization of non-Brownian, yet diffusive, particles. Here we present some analytic and numerical results for models of the ideal (low-density) limit in which the particles have no hydrodynamic or other interactions and hence undergo independent motions. We address three cases: sedimentation under gravity; confinement by a harmonic external potential; and rectification by a strip of 'funnel gates' which we model by a zone in which tumble rate depends on swim direction. We compare our results with recent experimental and simulation literature and highlight similarities and differences with the diffusive motion of colloidal particles.
\end{abstract}

Recent years have seen an upsurge of activity at the interface between physics and biology. In many cases, relatively wellestablished physics tools have been used to address problems of major interest to biologists. In other cases problems that might appear arcane to some (though not all) biologists have nonetheless posed interesting new physics questions. For instance, the motion of $E$. coli and similar bacteria involves a series of roughly straight-line 'runs' punctuated by rapid changes of direction ('tumbles') [1]. This run-and-tumble dynamics can, in idealized form, serve as a paradigm for non-Brownian diffusive motion, and be used to explore some central concerns of nonequilibrium dynamics, such as the origins of phase sepa+ ration in systems without detailed balance [3,4].

E. coli is a unicellular organism with external flagellar filaments, which form a bundle at one pole of the cell [1]. When these filaments synchronously rotate counterclockwise, the organism swims in trajectory that is basically straight (with small effects of rotational Brownian motion) [5]. A stochastic intracellular event results in a change of activity $[6,7]$ so that one or more filaments start rotating clockwise; the organism then tumbles $[8,9]$. The tumble is of short duration; once all filaments start rotating counterclockwise again, a new run begins.

Supposing the runs to have fixed direction and speed $v$, and the tumbles to occur instantaneously and randomly at rate $\alpha$ (with each tumble fully randomizing the direction of motion), it is easily shown that in free space such run-and-tumble organisms obey the diffusion equation at large length and time scales, with diffusivity $D=v^{2} / \alpha d$ where $d$ is the dimensionality. Yet, because bacteria are not Brownian particles close to thermal equilibrium, one cannot write the usual Einstein relations $D=k T \mu$. Here $\mu$ is the mobility, which controls the mean velocity $\mathbf{v}=\mu \mathbf{F}$ of a particle subject to an external force $\mathbf{F}$; whenever this force is conservative $(\mathbf{F}=-\nabla U)$, the Einstein relation ensures that, for truly Brownian particles, the equilibrium mean density $\rho$ obeys the Boltzmann distribution, $\rho \propto \exp [-\mu U / D]=\exp [-U / k T]$.

As will be seen from two of the examples below (sedimentation and the harmonic trap), forced run-and-tumble particles show more complicated behaviour than do Brownian ones. Indeed, even when forces are conservative and a flux-free steadystate density is recovered, this is not in general a Boltzmann distribution for the potential $U$, even if $D$ is rescaled at will: there is no general 'effective temperature' concept.

This was discussed briefly in [3] which however focussed on the case of interacting particles. In the present work we explore the noninteracting limit in more detail, presenting a range of results that include an exact steady-state calculation of the sedimentation decay length in 3D. (The interactions that we omit include hydrodynamics [10], which may bring important changes even at modest density.) Oddly, we have found very little discussion of external force fields in the previous literature on noninteracting run-and-tumble particles $[11,12]$, much of which assumes rotational symmetry of the set of possible particle velocities. This assumption is inspired by models of bacterial chemotaxis, which for good biological reasons take the tumble rate, and not the swim speed, to be a function of swim direction. But external forces, such as gravity, break this symmetry and thus require separate treatment.

As well as external force fields, we consider below the effect of asymmetric obstacles, such as funnel gates [13], arguing that these can be modelled by a zone in which there is effective asymmetry in the tumbling rates for particles moving in oppo- 
site directions. We show that this viewpoint provides a semiquantitative account of the rectification experiments of [13].

Effective temperature: We first consider noninteracting runand-tumble particles in 1D with symmetric tumbling rates $\alpha$ and swim speeds $v$. At times long compared to $\alpha^{-1}$ and length scales large compared to $\ell \equiv v / \alpha$ (the run-length) the motion is diffusive, with diffusivity $D=v^{2} / \alpha$. In the presence of an external potential $U$, the speeds of left- and right-going bacteria become $v_{L, R}=v \pm \mu \nabla U$. The diffusion-drift equation for the probability density $P$ becomes $[3,11]$

$$
\begin{aligned}
\partial_{t} P & =-\nabla \cdot J \\
J & =-\frac{v^{2}-\mu^{2}(\nabla U)^{2}}{\alpha} \nabla P-\mu \nabla U\left(1-\frac{2 \mu \Delta U}{\alpha}\right) P
\end{aligned}
$$

If the external field only slightly perturbates the swim speed of the bacterium, that is if $|\mu \nabla U| \ll v$, and if it does not induce large gradients of the velocity field on the scale of the run length $\left(\left|\frac{v \mu \Delta U}{\alpha} \ll v\right|\right)$, the current becomes at first order $J=-\frac{v^{2}}{\alpha} \nabla P-\mu(\nabla U) P$ and the flux-free steady state then obeys

$$
P \propto \exp [-\hat{\beta} U] ; \quad \hat{\beta}=\frac{\mu \alpha}{v^{2}}=\frac{\mu}{D}
$$

Such 1D bacteria thus behave as "hot colloids", with an effective temperature $1 / \hat{\beta}$; however this breaks down nonperturbatively [3]. We show below that the same is true for $d>1$.

Sedimentation: An obvious physics question concerns the sedimentation equilibrium of run-and-tumble bacteria: what is the steady-state probability density $P(z)$ to observe a bacterium of buoyant mass $m$ at a height $z$ above a hard wall, in the presence of a downward force $F=-m g$ ? A technical complication is that the singular forces arising at the wall have to be described (or replaced by a rule for modifying nearby trajectories). This will materially influence the profile in a proximal region whose height is set by the run length $v / \alpha$. For simplicity we here consider only the distal part of the profile at much larger $z$.

In $1 \mathrm{D}$ the problem can be solved by the methods of $[3,11]$ which developed diffusion-drift equations for the density that nonetheless exactly recover flux-free [11] or general [3] steady states. A route more readily generalized to $d>1$ instead addresses the following equations for steady-state probability densities which contain no approximation beyond those of the defining model:

$$
\begin{aligned}
P(z) d z & \equiv \sum_{c= \pm 1} P(c, z) d z \\
P(c, z) d z & =\frac{\alpha}{2} \int_{0}^{\infty} P\left(z_{i}(z, c, \tau)\right) d z_{i} e^{-\alpha \tau} d \tau
\end{aligned}
$$

Here $P(c, z)$ is the probability density of finding a bacterium at height $z$ that swims either upwards (labelled $c=1$ ) or downwards $(c=-1)$ and $P(z)$ is the total probability density. Eq. (4) then states that a particle of type $c$ currently (at $t=0$, say) in the small height interval $(z, z+d z)$ last underwent a tumble at some earlier time. (Note that in $1 \mathrm{D}$, half the tumbles leave $c$ unchanged, whence the $1 / 2$ factor). If this event occurred between times $t=-\tau$ and $t=-(\tau+d \tau)$, the bacterium was then in a height range $\left(z_{i}, z_{i}+d z_{i}\right)$ where $z_{i}=z-\left(v c-v_{T}\right) \tau$. Here $v_{T}=-\mu F$ so that $v c-v_{T}$ is the net upward velocity of a swimmer of type $c$. (Note that for constant $F$ only, we also have $d z_{i} / d z=1$.) Thus the contribution to $P(c, z) d z$ of the 'age-slice' $(\tau, \tau+d \tau)$ is $(\alpha / 2) d \tau \times P\left(z_{i}\right) d z_{i} \times \exp [-\alpha \tau]$ where the three factors represent respectively the probability of a tumble event occurring in the relevant time interval; the probability of finding a bacterium at this time in the required height range to later arrive at $z$; and the survival probability against further tumbles at intervening times. Integrating this over $\tau$ recovers (4).

Summing (4) over $c$ yields a linear integral equation for $P(z)$ which can be solved by Fourier transform. Defining $P(\omega)=$ $\int_{-\infty}^{\infty} P(z) \exp (-i \omega z) d z,(3)$ and (4) imply

$$
\begin{gathered}
P(\omega) \omega\left[\omega\left(v^{2}-v_{\tau}^{2}\right)-i \alpha v_{\tau}\right]=0 \\
\Rightarrow P(\omega)=A \delta(\omega)+B \delta\left(\omega-\frac{i \alpha v_{\tau}}{v^{2}-v_{\tau}^{2}}\right)
\end{gathered}
$$

Thanks to the flux-free boundary condition at $z=0$, one has $A=0$ and inverting the Fourier transform yields

$$
\begin{aligned}
P(z) & =P_{0} e^{-\kappa z} \\
\kappa & =\frac{v_{T} \alpha}{v^{2}-v_{T}^{2}}
\end{aligned}
$$

where we have assumed $v>v_{T}$. In the perturbative regime $v_{\tau} \ll v$, one recovers the result (2). More generally, however, although Eq. (7) has the Perrin form (a Boltzmann distribution under gravity) Eq. (8) contradicts the result $\kappa=v_{T} / D$ for Brownian particles. In particular, the sedimentation length $\kappa^{-1}$ tends to zero as $v_{T}$ is raised towards $v$, while for $v_{T}>v$ both species of particle have a net downward motion and nothing (short of true Brownian motion, if present) can save the system from complete gravitational collapse [3].

Unlike the differential equation methods of $[3,11]$, the above integral-equation approach generalizes, without further approximation, to the 3D case. By identical reasoning one finds an exact equation for sedimentation of ideal swimmers

$$
\begin{aligned}
P(z) d z & =2 \pi \int_{-1}^{1} d c P(c, z) d z \\
P(c, z) d z & =\frac{\alpha}{4 \pi} \int_{0}^{\infty} P\left(z_{i}(z, c, \tau) d z_{i} e^{-\alpha \tau} d \tau\right.
\end{aligned}
$$

where $c=\cos \theta$ is the angle between the propulsion direction and the vertical, so that the net upward velocity is $v c-v_{T}$ as before, and the definition of $z_{i}(z, c, \tau)$ is likewise unchanged. Integrating (4) over $c$ and proceeding as before via Fourier transform, one gets for $P(\omega)=\int_{-\infty}^{\infty} P(z) \exp (i \omega z)$

$$
P(\omega)=P(\omega) \frac{\alpha}{\omega v} \int_{0}^{\infty} \exp \left(-\frac{\alpha+i \omega v_{\tau}}{\omega v}\right) \frac{\sin (u)}{u} d u
$$

The r.h.s can be computed, for instance by power expansion of the sin function, to yield

$$
P(\omega)\left[\frac{\omega v}{\alpha}-\arctan \left(\frac{\omega v}{\alpha+i \omega v_{\tau}}\right)\right]=0
$$




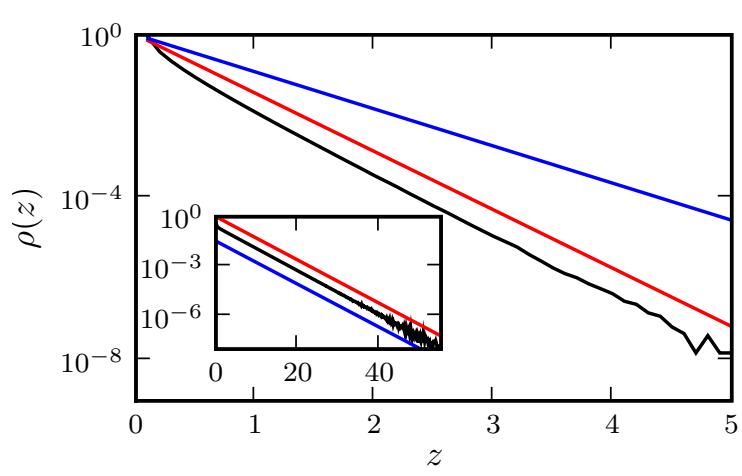

Figure 1: Simulations of 3D run-and-tumble bacteria under gravity. Black: full numerics; red: exact solution; blue: quasi-Brownian approximation, with vertical offsets introduced for clarity. For $v_{\tau} / v=$ 0.7 , the deviation from the diffusive approximation is apparent. Inset For $v_{\tau} / v=0.1$, the diffusive approximation works very well.

The constant solution $P(\omega)=\delta(\omega)$ is once again forbidden by the flux-free boundary condition and one is left with $P(\omega)=$ $\delta\left(\omega-\omega_{0}\right)$ where $\omega_{0} v / \alpha=\arctan \left(\omega_{0} v /\left(\alpha+i \omega_{0} v_{\tau}\right)\right)$. Inverting the Fourier transform again yields the Perrin form (7), where $\kappa=i \omega_{0}$ now has to satisfy

$$
\ln \left(\frac{\kappa\left(v_{T}+v\right)+\alpha}{\kappa\left(v_{T}-v\right)+\alpha}\right)=\frac{2 \kappa v}{\alpha}
$$

Just as in $1 \mathrm{D}$, linearization of (13) yields the quasi-Brownian result $\left(\kappa=v_{T} / D\right.$ with $\left.D=v^{2} / 3 \alpha\right)$ at leading order in $v_{T}$, whereas beyond this, although the Perrin form (7) is maintained, its decay rate $\kappa$ again diverges when $v_{T} \rightarrow v$ and is infinite for $v_{T}>v$. In this regime (barring true Brownian motion) gravitational collapse occurs just as found in $1 \mathrm{D}$, and for the same reason. Interestingly, since (13) and (8) differ, run-andtumble diffusion cannot generally be factorized over orthogonal spatial directions even if, as here, the forcing is uniaxial and translationally invariant.

For a typical bacterium in terrestrial gravity one expects $v_{T} \simeq 1 \mu \mathrm{ms}^{-1}$ whereas $v \sim 20 \mu \mathrm{ms}^{-1}$ [1]. This means that the sedimentation problem (at least in the distal region) remains close to the quasi-Brownian limit; however the opposite limit could easily be reached using a centrifuge. We hope that our exact 3D solution will encourage experiments on bacterial sedimentation. We have also tested our prediction by an explicit stochastic simulation of an ensemble of run-and-tumble particles, recovering satisfactorily the distal solution (Fig. 1).

Trapping: Another physics question of possible experimental relevance concerns trapped bacteria. Consider, e.g., a harmonic potential $\left(U(z)=\lambda z^{2} / 2 \mu\right)$ for which $v_{T}(z)=-\lambda z$. In 1D, (3 4) still hold, but solving $\dot{z}=v c-\lambda z$ gives in (4)

$$
\lambda z_{i}(z, c, \tau)=v c-(v c-\lambda z) \exp [\lambda \tau]
$$

so that $d z_{i} / d z=\exp [\lambda \tau]$. The solution found from the diffusion-drift formulation [3] is, for $|z|<v / k$,

$$
P(z)=P(0)\left[1-(\lambda z / v)^{2}\right]^{\alpha / 2 \lambda-1}
$$

with $P(z)=0$ for $|z|>v / \lambda$. On substitution in 34 with use of (14), we confirm (15) to be an exact 1D result.

As noted already in [3] it is not of Boltzmann form $(P \sim$ $\left.\exp \left[-\alpha \lambda z^{2} / 2 v^{2}\right]\right)$, nor can it be made so by any choice of "effective temperature' (any rescaling of $D$ ). As discussed earlier, it does however approach the quasi-Boltzmann result if simultaneously $(k \lambda / v)^{2} \ll 1$ (defining a region where $v_{T} \ll v$ ) and $\alpha / \lambda \gg 1$ (i.e. the velocity barely changes over a run length). This additional requirement ensures that tumbling is frequent on the length scale over which $v_{T}$ changes appreciably and that the diffusion-drift approximation (1) holds. (Note that our exact result (15) is not limited to this case.) The vanishing of $P$ for $|z|>v / \lambda$ arises, as in sedimentation, because in this region all particles are moving towards the centre of the trap and a finite density cannot be maintained in steady state.

The breakdown of the 'effective temperature' concept is much more severe here than for sedimentation (where the Perrin form was maintained, albeit with an altered $\kappa$ ). Whenever $\alpha / 2 \lambda$ is not large the distribution is strongly non-Gaussian; for $\alpha=2 \lambda$ it is flat and for $\alpha \leq 2 \lambda$ it is bimodal, with an accumulation of bacteria at the outer edges the trap (Fig. 2). This phenomenology has a simple intuitive explanation. First, let us note that the motion of right and left going bacteria amounts to gradient descent in two effective potentials $U_{L, R}=$ $\pm v z / \mu+\lambda z^{2} /(2 \mu)$ whose minima are $z_{R, L}= \pm v / \lambda$. The average duration of a run is $\alpha^{-1}$ whereas after reversal of a particle at $z=z_{R, L}$ it takes time $\ln 2 / \lambda \sim \lambda^{-1}$ to reach the centre. For $\alpha \gg \lambda$, a bacterium tumbles many times before it can cross the trap and it thus diffuses within the average, quadratic potential $\left(U_{R}+U_{L}\right) / 2$, giving a Gaussian steady state, $P \sim \exp \left[-\alpha k z^{2} / 2 v^{2}\right]$. On the other hand, when $\alpha \ll \lambda$, bacteria descend their potential to $z_{R}$ or $z_{L}$, spend a long time there and then tumble. This generates a bimodal distribution whose maxima are at $z_{R}$ and $z_{L}$.

Stochastic simulations of run-and-tumble particles in 3D indicate a qualitatively similar behaviour for a radial harmonic trap $\mathbf{v}_{T}=-\lambda \hat{\mathbf{r}}$ (Fig. 3). Although such a trap (with trapping radius large compared to a bacterium's diameter) is hard to realize experimentally [14], the same physical mechanism (with maximal particle density at the outer edge) would apply for bacteria trapped, say, in an emulsion droplet. It would be interesting to look experimentally for qualitative deviations from quasi-Boltzmann statistics in such cases. However, as we address elsewhere [15] coupling of the particles to a momentumconserving solvent could in some case drastically alter, or even destroy, the flux-free steady states predicted here, as could, on larger time scales, the birth and death of bacteria [16].

Rectification: An intriguing recent experiment has shown how to trap run-and-tumble bacteria by means quite different from the external force fields addressed above. This involves 'rectification' of the random swimming motion so that particles move preferentially into the trap [13]. Two rectangular microfluidic enclosures contain swimming bacteria (effectively in 2D) separated by a wall of funnels (See Fig. 4). Starting from a uniform density, the system evolves until at long times the two compartments have uniform populations of bacteria, but with different densities. The same experiment done with 


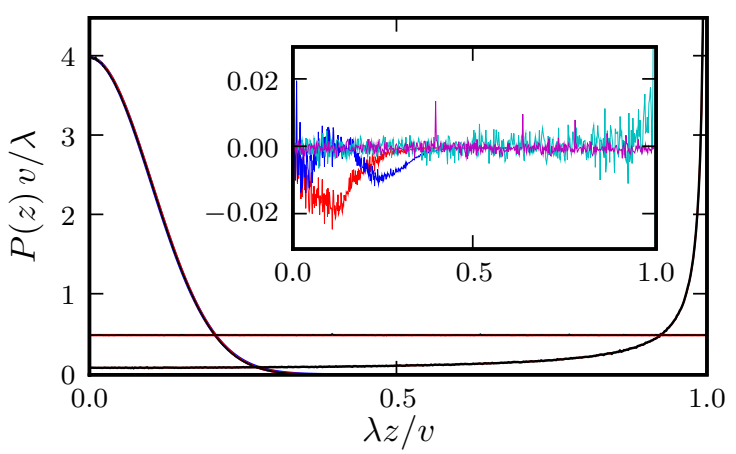

Figure 2: 1D run-and-tumble bacteria in a quadratic trap $(v=1, \alpha=$ $1)$. The result of the simulation of run-and-tumble dynamics is displayed in the perturbative case $(\lambda=0.01)$, the flat case $(\lambda=0.5)$ and the strong trapping regime $(\lambda=5)$. The later shows bimodality as predicted. Also plotted are the gaussian approximation in blue (for weak trapping) and the exact result in red (for all cases). They overlap perfectly with the numerics. Inset Differences between numerics and : in the weak trapping case, the exact result (red) and the gaussian approximation (blue); in the flat case, the exact result (magenta); in the strong trapping case, the exact result (cyan). The agreement is in all cases very good, the errors being roughly two order of magnitudes smaller that the densities.

non-swimming bacteria results in no rectification.

Our model neglects hydrodynamics interactions, both between bacteria and with the walls [10], and simply assumes that a bacterium swims along a wall after colliding with it, until its next tumble. (Such motion has been observed in this experiment [13] and may indeed be of hydrodynamic origin [17].) The above fully defines a stochastic model suitable for simulation (Fig. 4). To create an analytical approximation, we represent the wall interactions as an additional, non-stochastic, reorientation process ('forced tumbling') whose rate depends on the initial swim direction: while the two particles in Fig. 4 start symmetrically either side of the plane of the wall, one will cross this plane whereas the other will not. A wall of funnels can thus be modelled by a narrow strip in which (say) left-to-right tumbles are favoured over right-to-left ones.

We next show that this simplified model accounts semiquantitatively for the rectification observed in [13]. First consider a 1D system with densities $R(x), L(x)$ of right- and leftmoving particles of equal speed $v$ but that tumble with different rates $\alpha_{R, L}(x)$. Neglecting noise terms [3] these obey [11,12]

$$
\begin{aligned}
& \partial_{t} R=-v \nabla R-\frac{\alpha_{R}}{2} R+\frac{\alpha_{L}}{2} L \\
& \partial_{t} L=v \nabla L-\frac{\alpha_{L}}{2} L+\frac{\alpha_{R}}{2} R
\end{aligned}
$$

The $1 / 2$ factor in front of the tumbling rates formally account for the fact that after a tumble, bacteria choose their new direction at random; but any bias in outgoing trajectories can, in 1D, be absorbed into a redefinition of $\alpha_{R, L}$.

We now divide the system into three regions: for $x \in$ $[0, L-\epsilon] \cup[L+\epsilon, 2 L]$, bacteria tumble symmetrically with a constant rate $\alpha_{R, L}=\alpha_{o}$, whereas for $x \in[L-\epsilon, L+\epsilon]$ bacteria swimming to the left are converted into right-going bacteria

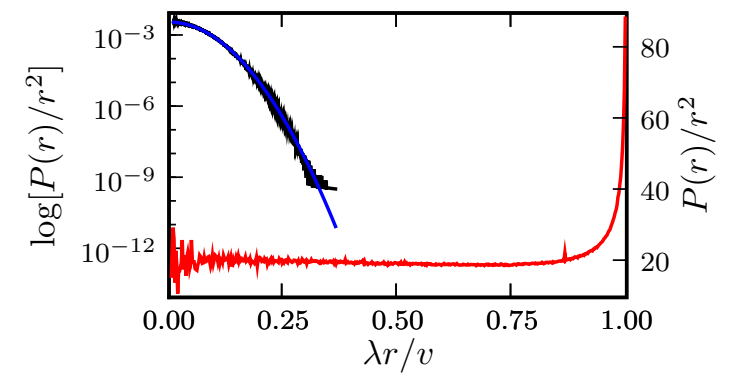

Figure 3: 3D run-and-tumble bacteria in a quadratic trap $(v=1, \alpha=$ 1). Left axis: weak trapping $(\lambda=0.01)$. Numerical results in black coincide with the Boltzmann-like approximation in blue $P(r) \simeq$ $r^{2} \exp \left(-3 \lambda r^{2} / 2\right)$. Right axis: strong trapping case $(\lambda=2$ in red), $P(r) / r^{2}$ increases near the edge of the trap. The bacteria accumulate on the surface of the sphere of radius $v / \lambda$.

with an additional rate $\alpha_{c}$, i.e. $\alpha_{L}=\alpha_{o}+2 \alpha_{c}, \alpha_{R}=\alpha_{o}$. The wall of funnels is thus replaced by a strip of width $2 \epsilon$ in which the sole effect is to create a bias in the tumble rates. Introducing the total probability density $P=R+L$ and current $J=v(R-L)$, Eqs. 16 are equivalent to the continuity equation (1) coupled to one for the current evolution [3,11]

$$
\frac{1}{\alpha} \partial_{t} J=-D \nabla P+V P-J
$$

with $\alpha \equiv\left(\alpha_{R}+\alpha_{L}\right) / 2 ; V \equiv v\left(\alpha_{L}-\alpha_{R}\right) / 2 \alpha$; and $D \equiv v^{2} / \alpha$.

In the left and right compartments, where $\alpha_{R}=\alpha_{L}$, the stationary solution of (1) and (17) is obviously provided by constant profiles, which we denote $\rho_{1}$ and $\rho_{2}$, respectively. In the intermediate strip region however, the solution is an exponential increase $\rho(x) \propto \exp \left[x \alpha_{c} / v\right]$. Matching these three solutions, one finds for the rectification ratio

$$
A_{1 D}=\frac{\rho_{2}}{\rho_{1}}=\exp \left[\frac{2 \alpha_{c} \epsilon}{v}\right]
$$

Allowing for the inverse dependence of $D$ on dimensionality, a pseudo-2D version of (18) would then yield

$$
A_{p 2 D}=\exp \left[\frac{4 \alpha_{c} \epsilon}{v}\right]
$$

In fact, a fully $2 \mathrm{D}$ analysis is also possible for a specific form of the tumbling bias. Specifically, if we take constant $v$ and a tumble rate $\alpha_{o}+\boldsymbol{\alpha}_{1}$.u for a swimmer moving along unit vector $\mathbf{u}$, then the density $\rho(\mathbf{x})$ obeys in flux-free steady state [11]

$$
\rho(\mathbf{x})=\rho(\mathbf{0}) \exp \left[-v^{-1} \int_{\mathbf{0}}^{\mathbf{x}} \alpha_{\mathbf{1}}\left(\mathbf{x}^{\prime}\right) d \mathbf{x}^{\prime}\right]
$$

Note that single-valuedness of the integral requires $\alpha_{1}=$ $-\nabla \phi$ for some scalar field $\phi$; without this, there is no fluxfree steady state [11]. For our problem, taking $\boldsymbol{\alpha}_{1}$ to be a constant vector normal to the plane of funnels within the strip and zero outside it, we obtain $A_{2 D}=\exp \left(2 \alpha_{1} \epsilon / v\right)$. Note that the chosen angular dependence of the tumbling rate does not 

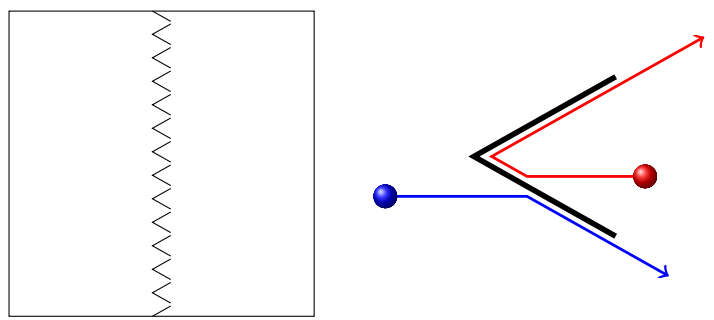

Figure 4: Left: The sides of the enclosures in [13] are $400 \mu \mathrm{m}$ long, the arms of the funnels are $27 \mu m$ long and their apex angle is $\pi / 3$, the funnels are separated by gaps $3.8 \mu \mathrm{m}$ wide. Right: After a collision with a wall, a particle tends to swim along it. The wall thus induces a tumble with fixed outgoing direction.

account precisely for what happens close to the funnel and we should thus not expect a perfect quantitative agreement with the experiment.

We now compare these theoretical predicitions with our direct stochastic simulations of $2 \mathrm{D}$ run-and-tumble particles in the geometry described previously. We simulate a square chamber $400 \mu \mathrm{m}$ wide similar to the one used in [13] (Fig. 4, with each funnel arm $27 \mu \mathrm{m}$ long and the gaps $3.8 \mu \mathrm{m}$ wide. The apical angle is $\pi / 3$. In the simulations, when a swimmer hit a wall, it aligns with it and proceeds in that direction until the next tumble. This indeed leads to rectification (Fig. 5), but to compare quantitatively with our theoretical model, we must measure the effective tumbling asymmetry $\alpha_{c}$ or $\boldsymbol{\alpha}_{1}$.

Within the 1D (or pseudo-2D) approach a lower bound for $\alpha_{c}$ is provided by the frequency at which particles swimming to the left encounter the apices of the funnels. Numerically, this can be obtained as $\alpha_{c}=n_{c o l} / t_{L}^{\epsilon}$, where one follows a very long trajectory and measures both $n_{c o l}$, the total number of such apical collisions, and $t_{L}^{\epsilon}$, the time spent swimming leftwards within an enclosing strip of size $2 \epsilon$. We then smoothly increased $\epsilon$ until $\epsilon \alpha_{c}$ converges. In the fully 2D analysis, integrating the tumble rate $\alpha_{o}+\boldsymbol{\alpha}_{1}$.u over each hemisphere gives $\left\langle\alpha_{L, R}\right\rangle=\alpha_{o} \pm 2 \alpha_{1} / \pi$ with only half these tumbles changing the horizontal direction of swimmers. The corresponding lower bound is then provided by $\alpha_{1}=\pi n_{\text {col }} /\left(2 t_{L}^{\epsilon}\right)$. The comparisions between numerics and theory (with these limiting parameter values) are presented in Fig. 5. Increasing the swim-speed increases the flux of bacteria colliding with the funnels, and thus raises both $n_{c o l} / t_{L}^{\epsilon}$ and the rectification ratio. The agreement with theory is semiquantitative, and quite satisfactory given the simplicity of the models. A more detailed analysis of the angular dependence of the tumbling rate could probably improve the quantitative agreement, but might not add much further physical insight.

The 'forced-tumbling' picture of rectification admits several parameter-free predictions. Specifically, the rectification ratio depends only on the local properties of the funnel wall (gap size, apical angle, etc.) and not its overall extent or orientation relative to the walls of the chambers; the size and shape of these chambers are themselves irrelevant so long as they are large
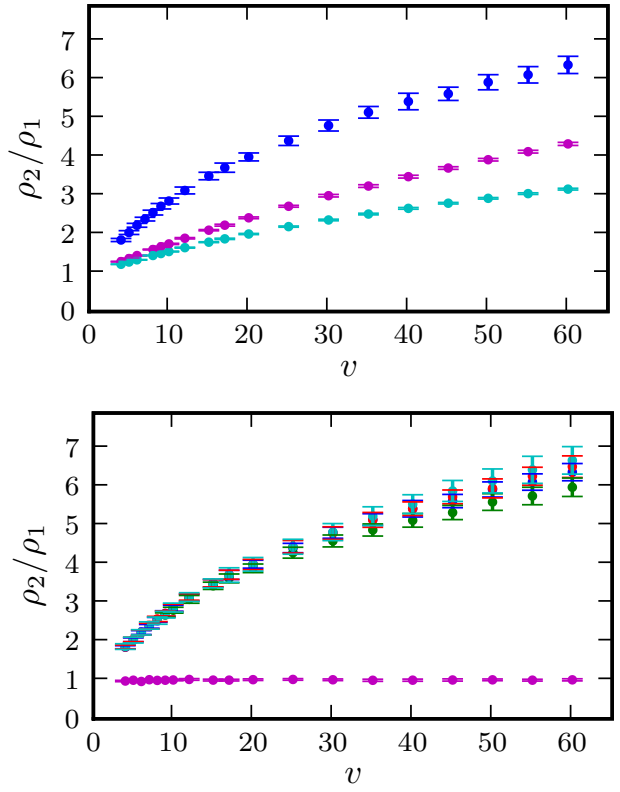

Figure 5: Comparision between theory and simulations (setting $\alpha_{o}=$ $1 s^{-1}$ for the chamber shown in Fig. 4). Top The rectification ratio $A$ measured from numerics (blue, top line), for velocities ranging from $4 \mu \mathrm{m} \mathrm{s}^{-1}$ to $60 \mu \mathrm{m} \mathrm{s}^{-1}$, is compared with $A_{p 2 d}$ (magenta, middle) and $A_{2 d}$ (cyan, bottom). The experimental value for the ratio is around 3 [13], and is reproduced here for $v \simeq 15 \mu \mathrm{m} \mathrm{s}^{-1}$. In the experiment, the velocity was measured to be $v=20 \pm 5 \mu \mathrm{m} \mathrm{s}^{-1}$ and the tumbling rate $\alpha_{o}=0.75 \pm 0.25 \mathrm{~s}^{-1}$ [18]. Bottom As predicted by our theory, doubling the system width (from $L=400 \mu \mathrm{m}$ in red to $L=800 \mu \mathrm{m}$ in blue) does not change the ratio. When doubling the height (to $H=800 \mu \mathrm{m}$ in green) and the number of funnels (from 13 to 26), the curves overlap until the velocity is large enough to reveal finite size effects. An asymetric chamber $(L=600 \mu \mathrm{m}$ with the right cavity twice as wide as the left one) also yields overlapping results (cyan). Last, bacteria colliding elastically with the walls are not rectified (magenta).

compared to the run length. (This all follows from the path invariance of the integral in 20].) Some simple checks of this (doubling the chamber width or height or using an asymetric chamber) are made in Fig. 5

Detailed balance and symmetry breaking: Our stochastic simulations differ significantly from those of Wan et al. [19] who simulated a similar geometry. First, these authors assumed the tumbling of bacteria to occur at regular rather than Poissonian time intervals. Our drift-diffusion picture shows this to be unimportant; this is fortunate since tumbling events for real bacteria are thought to be near-Poissonian $[1,2]$. Second, Wan et al. represented each funnel by a force field deriving from a crescent-shaped potential barrier. This force field can overcome the swimming force, but cannot change its direction, whereas the latter is the key element captured by our own 'forced tumbling' description. Omission of this effect effect may explain why, to achieve the experimental value of $A \simeq 3$ [13], these authors invoked a run-length $v / \alpha_{o}$ not merely larger than the funnel size but comparable to the size $L$ of their entire chamber. (In this limit, almost every run event encounters the wall 
of funnels whereas in the experiment only runs starting within a strip of width $v / \alpha_{o} \ll L$ do so.) Our own simulations instead achieve the observed $A \simeq 3$ with experimentally plausible parameters $\left(v \sim 15 \mu \mathrm{ms}^{-1}, \alpha_{o}=1 \mathrm{~s}^{-1}\right)$.

More fundamentally, it has long been known that to produce directed motion out of fluctuations (ratchet effect), one needs to break both space-inversion and time-reversal symmetries [20]. The spatial symmetry breaking is of course provided by the asymmetric shape of the funnels themselves; for a wall perforated by symmetric channels, no rectification can arise [13]. Time reversal symmetry is more subtle, however. Whereas the motor force of bacteria does break this symmetry, at the coarsegrained (diffusion-drift) level, detailed balance is perfectly restored (albeit with respect to a non-Boltzmann distribution) in both the sedimentation and trap examples dealt with above. We therefore contend that it is actually the collisions of bacteria with the funnel walls that break the time-reversal symmetry in the sense required to create a 'ratchet' from the funnel geometry (Fig. 4). To check this, we simulated the same 2D funnel system, with full run-and-tumble dynamics, in a case where the entrainment of swimmers upon encountering a wall is replaced by a simple elastic collision law. Notably, no rectification whatever is observed in this case (Fig. 5).

Insofar as the rectification problem is subject to Eq20, (see below for exceptions) its steady states are again subject to detailed balance once the diffusion-drift coarse graining is applied. The dynamics of approach to states states can be addressed within the diffusive approximation by neglecting $\dot{J}$ in (17). This yields a Fokker-Planck equation whose late-time relaxation is governed by the first excited state, which for large systems is a diffusive mode with relaxation time of order of $\tau=L^{2} / D$. For a velocity of $15 \mu \mathrm{m} \mathrm{s}^{-1}$ in the geometry of Galajda et al., this gives a time scale of the order of 10 minutes, which is quite consistent with the exponential convergence measured in [13].

Steady state fluxes: The restriction $\boldsymbol{\alpha}_{1}(\mathbf{x})=-\nabla \phi$ encountered in connection with 20 is necessary for steady states in which the flux vanishes everywhere. It is easy to envisage experiments that violate this criterion, for instance if walls of uniform funnel density but both inward and outward orientations are used to create a closed shape. If the outward-rectifying sections comprise only a small part of the perimeter (the exact criterion may depend on shape) the mean density within the trap will still be higher than outside. Yet, if any such sections are present, these will carry a steady outward flux, balanced by an influx elsewhere. Thus the steady state contains circulating currents. This does not seem to have been looked for in [13].

Conclusion: We have calculated and discussed in this letter several steady-state density distributions within an idealized model [11] of noninteracting run-and-tumble bacteria; our results included a new exact solution for 3D sedimentation. For particles in harmonic traps the mechanism causing extremely non-gaussian density profiles at large ratios of run-length to trap size was elucidated. The phenomenon of rectification of run-and-tumble bacteria by funnel walls was addressed using an asymmetric tumble rate within both a 1D and 2D model, giving semiquantitative agreement with direct stochastic simu- lations and, using plausible parameters, with the experiments of [13]. We found no rectification in the case where each particle collides elastically with a funnel wall, rather than swimming parallel to the wall until its next tumble. This suggests, at odds with [19], that wall interactions (rather than the intrinsic asymmetry of bacterial propulsion mechanisms) are the fundamental source of time-reversal asymmetry at the coarse-grained level relevant to the rectification experiments of [13].

Acknowledgments: We thank R. Austin, R. Blythe, P. Galajda, J. Kurchan, J.-F. Joanny, S. Ramaswamy and R. Nash for discussions and EPSRC (EP/E030173 and GR/T11753) for funding. MEC holds a Royal Society Research Professorship.

\section{References}

[1] H. C. Berg, E. coli in Motion, Springer, NY (2004)

[2] H.C. Berg and D.A. Browm, Nature 239, 500 (1972)

[3] J. Tailleur, M. E. Cates, Phys. Rev. Lett. 100, 218103 (2008)

[4] R. A. Blythe, M. R. Evans, J. Phys. A 40, R333-R441 (2007)

[5] H.C. Berg and R.A. Anderson, Nature 245 380-382 (1973)

[6] D. Bray, Cell movements, 2nd edn. Garland, NY (2001)

[7] D. Webre, P. Wolanin and J. Stock, Curr. Biol. 13, R47-R49 (2003)

[8] S.H.R. Larsen, et al., Nature 249, $74-77$ (1974)

[9] L. Turner, W.S. Ryu and H.C. Berg, J. Bacteriol. 182, 2793-2801 (2000)

[10] A. Sokolov, I. S. Aranson, J. O. Kessler and R. E. Goldstein, Phys. Rev. Lett. 98, 158102 (2007); C. Dombrowski, et al., Phys. Rev. Lett. 93, 098103 (2004); T. Ishikawa and T. J. Pedley, Phys. Rev. Lett. 100, 088103 (2008)

[11] M. J. Schnitzer, Phys. Rev. E 48, 2553-2568 (1993)

[12] T. Hillen and H. G. Othmer, SIAM J. Appl. Math. 61, 751-775 (2000); H. G. Othmer and T. Hillen, SIAM J. Appl. Math. 62, 1222-1250 (2002); R. Erban and H. G. Othmer, SIAM J. Appl. Math. 65, 361-391 (2005)

[13] P. Galajda, J. Keymer, P. Chaikin, R. Austin, J. Bacteriol. 189, p. 8704-8707 (2007); P. Galajda,et al., J. Modern Optics 55, 3413$3422(2008)$

[14] S. Chattopadhyay, R. Moldovan, C. Yeung, X.L. Wu, Proc. Natl. Acad. Sci. USA 103, 13712 (2006)

[15] R. Nash, et al., work in progress.

[16] C. Barrett-Freeman, M. R. Evans, D. Marenduzzo, and W. C. Poon, Phys. Rev. Lett. 101, 100602 (2008)

[17] A.P. Berke, L. Turner, H.C. Berg, E. Lauga, Phys. Rev. Lett. 101, 038102 (2008)

[18] P. Galajda, private communication

[19] M.B. Wan, C.J. Olson Reichhardt, Z. Nussinov, C. Reichhardt, Phys. Rev. Lett. 101, 018102 (2008)

[20] M.O. Magnasco, Phys. Rev. Lett. 71, 1477 (1993) J. Prost, J.F. Chauwin, L. Peliti and A. Ajdari, Phys. Rev. Lett. 72,2652 (1994); 